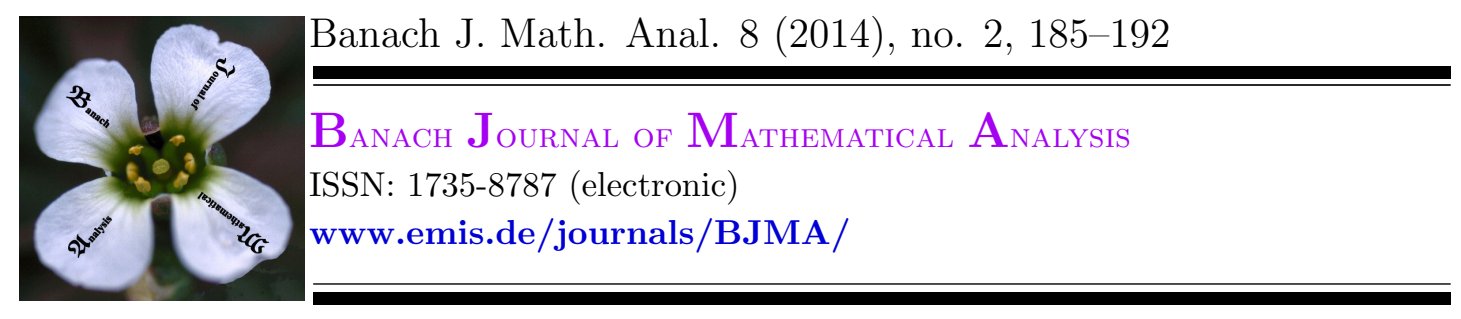

\title{
JORDAN-VON NEUMANN CONSTANT FOR BANAŚ-FRA̧CZEK SPACE
}

\author{
CHANGSEN YANG
}

Communicated by Q. Xu

\begin{abstract}
For any $\lambda \geq 1, \mathbb{R}_{\lambda}^{2}$ is Banaś-Frączek space, the exact value of the Jordan-von Neumann constant $C_{N J}\left(\mathbb{R}_{\lambda}^{2}\right)$ is investigated. By careful calculations, $C_{N J}\left(\mathbb{R}_{\lambda}^{2}\right)=2-\frac{1}{\lambda^{2}}$ is given.
\end{abstract}

\section{INTRODUCTION}

In order to study the geometric structure of Banach spaces, many recent studies have focused on the Jordan-von Neumann (NJ)constant. It is proved that the NJ constant is strongly connected with some geometric structures, such as uniform non-squareness and uniform normal structure. Hence many papers have appeared for how to compute the NJ constant.

Throughout this paper, $X$ is a nontrivial Banach space. We will use $B_{X}, S_{X}$ and $e x\left(B_{X}\right)$ to denote the unit ball, unit sphere of $X$ and the set of extreme points of $B_{X}$, respectively.

Recall that the Jordan-von Neumann (NJ) constant of a Banach space $X$ was introduced by Clarkson [2] as the smallest constant $\mathrm{C}$ for which

$$
\frac{1}{C} \leq \frac{\|x+y\|^{2}+\|x-y\|^{2}}{2\left(\|x\|^{2}+\|y\|^{2}\right)} \leq C
$$

holds for all $x, y \in X$ with $(x, y) \neq(0,0)$.

Date: Received: Sep. 27, 2013; Accepted: Dec. 22, 2013.

2010 Mathematics Subject Classification. Primary 46B20; Secondary 46 B99.

Key words and phrases. Jordan-von Neumann constant, modulus of convexity, BanaśFrączek space, James constant, uniform normal structure. 
An equivalent definition of the Jordan-von Neumann constant is found in [4] as the following form:

$$
C_{N J}(X)=\sup \left\{\frac{\|x+y\|^{2}+\|x-y\|^{2}}{2\left(\|x\|^{2}+\|y\|^{2}\right)}: x \in S_{X}, y \in B_{X}\right\} .
$$

We know that (see [9]) the Jordan-von Neumann constant $C_{N J}(X)$ can be computed by

$$
C_{N J}(X)=\sup \left\{\frac{\gamma_{X}(t)}{1+t^{2}}: 0 \leq t \leq 1\right\}
$$

where $\gamma_{X}(t)=\sup \left\{\frac{\|x+t y\|^{2}+\|x-t y\|^{2}}{2}: x, y \in S_{X}\right\}$.

We also note that $\gamma_{X}(t)=\sup \left\{\frac{\|x+t y\|^{2}+\|x-t y\|^{2}}{2}: x, y \in \operatorname{ex}\left(B_{X}\right)\right\}$ for a finite dimensional Banach space $X$. Therefore, for a finite dimensional Banach space $X$

$$
C_{N J}(X)=\sup \left\{\frac{\|x+t y\|^{2}+\|x-t y\|^{2}}{2\left(1+t^{2}\right)}: x, y \in \operatorname{ex}\left(B_{X}\right), 0 \leq t \leq 1\right\} .
$$

The modulus of convexity of a Banach space $X$ is defined for $\varepsilon \in[0,2]$ as

$$
\delta_{X}(\epsilon)=\inf \left\{1-\frac{\|x+y\|}{2}, x, y \in S_{X},\|x-y\| \geq \varepsilon\right\} .
$$

The function $\delta_{X}(\epsilon)$ is continuous on $[0,2)$ and strictly increasing on $\left[\epsilon_{0}(X), 2\right]$, where $\epsilon_{0}(X)=\sup \left\{\epsilon \in[0,2], \delta_{X}(\epsilon)=0\right\}$ is the characteristic of convexity of $X$. The non-square or James constant is defined as

$$
J(X)=\sup \left\{\min (\|x+y\|,\|x-y\|), x, y \in S_{X}\right\} .
$$

In $[6,7,8]$, an important relationship between the James constant and the NJ constant is given as follows,

$$
C_{N J}(X) \leq J(X)
$$

In [1], the authors considered the spaces $\mathbb{R}_{\lambda}^{2}:=\left(\mathbb{R}^{2},\|\cdot\|_{\lambda}\right)$, where $\lambda>1$ and

$$
\|(a, b)\|=\max \left\{\lambda|a|, \sqrt{a^{2}+b^{2}}\right\} .
$$

They asserted that

$$
\delta_{\mathbb{R}_{\lambda}^{2}}(\varepsilon)= \begin{cases}0 & \text { for } 0 \leq \varepsilon \leq 2 \sqrt{1-\frac{1}{\lambda^{2}}}, \\ 1-\lambda \sqrt{1-\frac{\varepsilon^{2}}{4}} & \text { for } 2 \sqrt{1-\frac{1}{\lambda^{2}} \leq \varepsilon \leq \frac{2 \lambda}{\sqrt{1+\lambda^{2}}},} \\ 1-\sqrt{1-\frac{\varepsilon^{2}}{4 \lambda^{2}}} & \text { for } \frac{2 \lambda}{\sqrt{1+\lambda^{2}}} \leq \varepsilon \leq 2\end{cases}
$$

Thus, the James constant $J(X)$ can be easily computed as $J(X)=\frac{2 \lambda}{\sqrt{1+\lambda^{2}}}$ by the modulus of convexity $\delta_{\mathbb{R}_{\lambda}^{2}}(\varepsilon)$ and the following formula [3]

$$
J(X)=\sup \left\{\varepsilon \in(0,2): \delta_{X}(\varepsilon) \leq 1-\frac{\varepsilon}{2}\right\} .
$$

Naturally we want to ask "what is its NJ constant?" In this note, we prove that $C_{N J}\left(\mathbb{R}_{\lambda}^{2}\right)=2-\frac{1}{\lambda^{2}}$, for any $\lambda \geq 1$. 


\section{MAin RESUlts}

The following theorem is our main result in this paper.

Theorem 2.1. Let $\lambda \geq 1$ and $\mathbb{R}_{\lambda}^{2}$ is the Banaś-Fraczek space. Then,

$$
C_{N J}\left(\mathbb{R}_{\lambda}^{2}\right)=2-\frac{1}{\lambda^{2}}
$$

In order to prove this theorem, first we give the following lemmas.

Lemma 2.2. If $\lambda \geq \sqrt{2}$ and $\left|x_{1}\right| \leq \frac{1}{\lambda},\left|y_{1}\right| \leq \frac{1}{\lambda}$, then

$$
\left(\lambda^{2}-1\right)\left|x_{1} y_{1}\right|+\sqrt{1-x_{1}^{2}} \sqrt{1-y_{1}^{2}} \leq 2-\frac{2}{\lambda^{2}} .
$$

Proof. By $\left|x_{1}\right| \leq \frac{1}{\lambda}$ and $\left|y_{1}\right| \leq \frac{1}{\lambda}$, we have

$$
\left(\lambda^{2}-2\right)\left|x_{1} y_{1}\right| \leq \frac{\lambda^{2}-2}{\lambda^{2}}
$$

that is

Therefore

$$
1-\left|x_{1} y_{1}\right| \leq\left(\lambda^{2}-1\right)\left(\frac{2}{\lambda^{2}}-\left|x_{1} y_{1}\right|\right)
$$

$$
\sqrt{1-x_{1}^{2}} \sqrt{1-y_{1}^{2}} \leq 1-\left|x_{1} y_{1}\right| \leq\left(\lambda^{2}-1\right)\left(\frac{2}{\lambda^{2}}-\left|x_{1} y_{1}\right|\right) .
$$

Thus, (2.1) is valid.

Lemma 2.3. Let $0 \leq \tau \leq 1,1<\lambda<\sqrt{2}$ and $0 \leq x_{1}, y_{1} \leq \frac{1}{\lambda}$. If $F\left(x_{1}, y_{1}\right)=$ $\lambda^{2}\left(x_{1}^{2}+\tau^{2} y_{1}^{2}\right)+2 \tau x_{1} y_{1}\left(\lambda^{2}-1\right)+2 \tau \sqrt{1-x_{1}^{2}} \sqrt{1-y_{1}^{2}}$, then

$$
\begin{aligned}
& \max \left\{F\left(x_{1}, y_{1}\right): 0 \leq x_{1}, y_{1} \leq \frac{1}{\lambda}\right\} \\
& =\max \left\{F\left(x_{1}, y_{1}\right):\left(x_{1}, y_{1}\right) \in \partial\left\{\left[0, \frac{1}{\lambda}\right] \times\left[0, \frac{1}{\lambda}\right]\right\}\right\},
\end{aligned}
$$

where $\partial\left\{\left[0, \frac{1}{\lambda}\right] \times\left[0, \frac{1}{\lambda}\right]\right\}$ denotes the boundary of the square $\left[0, \frac{1}{\lambda}\right] \times\left[0, \frac{1}{\lambda}\right]$.

Proof. Assume that $\tau \in(0,1]$. Suppose that $\max \left\{F\left(x_{1}, y_{1}\right): 0 \leq x_{1}, y_{1} \leq \frac{1}{\lambda}\right\}$ attains at some $\left(x_{1}, y_{1}\right) \in\left(0, \frac{1}{\lambda}\right) \times\left(0, \frac{1}{\lambda}\right)$, then $F_{x_{1}}\left(x_{1}, y_{1}\right)=F_{y_{1}}\left(x_{1}, y_{1}\right)=0$. And this implies

$$
\lambda^{2}+\tau\left(\lambda^{2}-1\right) \frac{y_{1}}{x_{1}}=\tau \sqrt{\frac{1-y_{1}^{2}}{1-x_{1}^{2}}},
$$

and

$$
\tau \lambda^{2}+\left(\lambda^{2}-1\right) \frac{x_{1}}{y_{1}}=\sqrt{\frac{1-x_{1}^{2}}{1-y_{1}^{2}}} .
$$

Now, through (2.2) multiplied by (2.3), then we have

$$
\tau=\tau \lambda^{4}+\lambda^{2}\left(\lambda^{2}-1\right) \frac{x_{1}}{y_{1}}+\tau^{2} \lambda^{2}\left(\lambda^{2}-1\right) \frac{y_{1}}{x_{1}}+\tau\left(\lambda^{2}-1\right)^{2} .
$$


But (2.4) is equivalent to

$$
0=2 \tau \lambda^{2}+\lambda^{2} \frac{x_{1}}{y_{1}}+\tau^{2} \lambda^{2} \frac{y_{1}}{x_{1}}
$$

and which is a contradiction.

Lemma 2.4. (i) If $1 \leq \lambda \leq \sqrt{2}$ and $0 \leq z \leq \frac{1}{\lambda^{2}}$, then

$$
9 \lambda^{4} z^{2}+48 z+25-34 \lambda^{2} z-8 \lambda^{2} z^{2}-\frac{16}{\lambda^{2}} z+\frac{16}{\lambda^{4}}-\frac{40}{\lambda^{2}} \geq 0 .
$$

(ii) If $0 \leq y \leq \frac{1}{\lambda}$ and $1 \leq \lambda \leq \sqrt{2}$, then

$$
2 y \sqrt{\lambda^{2}-1} \sqrt{1-y^{2}} \leq 5+2 y^{2}-3 \lambda^{2} y^{2}-\frac{4}{\lambda^{2}} .
$$

Proof. (i) Now, by letting $f(z)=9 \lambda^{4} z^{2}+48 z+25-34 \lambda^{2} z-8 \lambda^{2} z^{2}-\frac{16}{\lambda^{2}} z+\frac{16}{\lambda^{4}}-\frac{40}{\lambda^{2}}$, then we have

$$
f^{\prime}(z)=18 \lambda^{4} z+48-34 \lambda^{2}-16 \lambda^{2} z-\frac{16}{\lambda^{2}} .
$$

By $f^{\prime \prime}(z)=18 \lambda^{4}-16 \lambda^{2}>0$, we have

$$
f^{\prime}(z) \leq f^{\prime}\left(\frac{1}{\lambda^{2}}\right)=32-16\left(\lambda^{2}+\frac{1}{\lambda^{2}}\right) \leq 0 .
$$

Thus, $f(z) \geq f\left(\frac{1}{\lambda^{2}}\right)=0$.

(ii) Because (2.5) is equivalent to

$4 y^{2}\left(\lambda^{2}-1\right)\left(1-y^{2}\right) \leq 25+4 y^{4}+20 y^{2}-\left(10+4 y^{2}\right)\left(3 \lambda^{2} y^{2}+\frac{4}{\lambda^{2}}\right)+9 \lambda^{4} y^{4}+\frac{16}{\lambda^{4}}+24 y^{2}$,

that is

$$
34 \lambda^{2} y^{2}+8 \lambda^{2} y^{4}+\frac{16}{\lambda^{2}} y^{2}+\frac{40}{\lambda^{2}} \leq 9 \lambda^{4} y^{4}+\frac{16}{\lambda^{4}}+48 y^{2}+25
$$

Now, by letting $z=y^{2}$, we can obtain (2.5) by (i).

Lemma 2.5. (i) Let $A, B>0$ and $\tau \in[0,1]$, then

$$
\max \left\{\frac{A+B \tau}{2\left(1+\tau^{2}\right)}\right\} \leq \frac{A+\sqrt{A^{2}+B^{2}}}{4}
$$

(ii) Let $1<\lambda \leq \sqrt{2}, 0 \leq y \leq \frac{1}{\lambda}$ and $\tau \in[0,1]$, then

$$
\frac{1}{2}+\frac{1+\lambda^{2} \tau^{2} y^{2}+\frac{2 \tau}{\lambda}\left[\left(\lambda^{2}-1\right) y+\sqrt{\lambda^{2}-1} \sqrt{1-y^{2}}\right]}{2\left(1+\tau^{2}\right)} \leq 2-\frac{1}{\lambda^{2}}
$$

Proof. (i) Obviously, the function $g(\tau) \equiv \frac{A+B \tau}{2\left(1+\tau^{2}\right)}$ attains its maximum at $\tau=$ $\frac{\sqrt{A^{2}+B^{2}}-A}{B}$, hence (2.6) is valid. 
(ii) By use of (2.6), we have

$$
\begin{aligned}
& \frac{1}{2}+\frac{1+\lambda^{2} \tau^{2} y^{2}+\frac{2 \tau}{\lambda}\left[\left(\lambda^{2}-1\right) y+\sqrt{\lambda^{2}-1} \sqrt{1-y^{2}}\right]}{2\left(1+\tau^{2}\right)} \\
& =\frac{1+\lambda^{2} y^{2}}{2}+\frac{1-\lambda^{2} y^{2}+\frac{2 \tau}{\lambda}\left[\left(\lambda^{2}-1\right) y+\sqrt{\lambda^{2}-1} \sqrt{1-y^{2}}\right]}{2\left(1+\tau^{2}\right)} \\
& \leq \frac{3+\lambda^{2} y^{2}}{4}+\frac{\sqrt{\left(1-\lambda^{2} y^{2}\right)^{2}+\frac{4}{\lambda^{2}}\left[\left(\lambda^{2}-1\right) y+\sqrt{\lambda^{2}-1} \sqrt{1-y^{2}}\right]^{2}}}{4}
\end{aligned}
$$

So, we only need to prove the following inequality

$$
\sqrt{\left(1-\lambda^{2} y^{2}\right)^{2}+\frac{4}{\lambda^{2}}\left[\left(\lambda^{2}-1\right) y+\sqrt{\lambda^{2}-1} \sqrt{1-y^{2}}\right]^{2}} \leq 5-\frac{4}{\lambda^{2}}-\lambda^{2} y^{2} .
$$

Now, (2.8) is equivalent to the following inequality

$$
\begin{aligned}
& \left(1-\lambda^{2} y^{2}\right)^{2}+\frac{4}{\lambda^{2}}\left[\left(\lambda^{2}-1\right) y+\sqrt{\lambda^{2}-1} \sqrt{1-y^{2}}\right]^{2} \\
& \leq\left[\left(1-\lambda^{2} y^{2}\right)+4\left(1-\frac{1}{\lambda^{2}}\right)\right]^{2} .
\end{aligned}
$$

(2.9) can be changed into

$$
\frac{4}{\lambda^{2}}\left[\sqrt{\lambda^{2}-1} y+\sqrt{1-y^{2}}\right]^{2} \leq \frac{16\left(\lambda^{2}-1\right)}{\lambda^{4}}+\frac{8}{\lambda^{2}}\left(1-\lambda^{2} y^{2}\right) .
$$

By a simple computation, we have that (2.10) is equivalent to (2.5).

Lemma 2.6. Let $1<\lambda \leq \sqrt{2}, \tau \in[0,1]$ and $0 \leq y \leq \frac{1}{\lambda}$, then

$$
\frac{1}{2}+\frac{\lambda^{2} \tau^{2} y^{2}+2 \tau \sqrt{1-y^{2}}}{2\left(1+\tau^{2}\right)} \leq 2-\frac{1}{\lambda^{2}}
$$

and

$$
\frac{1}{2}+\frac{\lambda^{2} y^{2}+2 \tau \sqrt{1-y^{2}}}{2\left(1+\tau^{2}\right)} \leq 2-\frac{1}{\lambda^{2}} .
$$

Proof. Obviously, (2.12) implies (2.11).

Now, we prove $(2.12)$. By $\lambda^{2}\left(1-y^{2}\right)+\frac{\tau^{2}}{\lambda^{2}} \geq 2 \tau \sqrt{1-y^{2}}$, we have $\lambda^{2} y^{2}+$ $2 \tau \sqrt{1-y^{2}} \leq \lambda^{2}+\frac{\tau^{2}}{\lambda^{2}}$. Hence

$$
\frac{1}{2}+\frac{\lambda^{2} y^{2}+2 \tau \sqrt{1-y^{2}}}{2\left(1+\tau^{2}\right)} \leq \frac{1}{2}+\frac{\lambda^{2}+\frac{\tau^{2}}{\lambda^{2}}}{2\left(1+\tau^{2}\right)} \leq \frac{1}{2}+\frac{\lambda^{2}}{2} \leq 2-\frac{1}{\lambda^{2}},
$$

where the last inequality is obtained by $\left(\lambda^{2}-1\right)\left(\lambda^{2}-2\right) \leq 0$.

\section{Proof of Theorem2.1}

Assume that $\lambda>1$. Note that $\operatorname{ex}\left(B_{X}\right)=\left\{\left(z_{1}, z_{2}\right): z_{1}^{2}+z_{2}^{2}=1,\left|z_{1}\right| \leq \frac{1}{\lambda}\right\}$. 
Now we prove that

$$
\frac{\|x+\tau y\|^{2}+\|x-\tau y\|^{2}}{2\left(1+\tau^{2}\right)} \leq 2-\frac{1}{\lambda^{2}}
$$

holds for any $x, y \in e x\left(B_{X}\right)$ and any $\tau \in[0,1]$.

Case(I). $\lambda \geq \sqrt{2}$. Letting $x=\left(x_{1}, x_{2}\right), y=\left(y_{1}, y_{2}\right)$, then we have the following three cases.

Ia). If $\|x+\tau y\|_{2} \leq\left|\lambda\left(x_{1}+\tau y_{1}\right)\right|$ and $\|x-\tau y\|_{2} \leq\left|\lambda\left(x_{1}-\tau y_{1}\right)\right|$, then

$$
\begin{aligned}
\|x+\tau y\|^{2}+\|x-\tau y\|^{2} & =\lambda^{2}\left[\left(x_{1}+\tau y_{1}\right)^{2}+\left(x_{1}-\tau y_{1}\right)^{2}\right] \\
& =2 \lambda^{2}\left(x_{1}^{2}+\tau^{2} y_{1}^{2}\right) \leq 2\left(1+\tau^{2}\right) .
\end{aligned}
$$

Ib). If $\|x+\tau y\|_{2}>\left|\lambda\left(x_{1}+\tau y_{1}\right)\right|$ and $\|x-\tau y\|_{2}>\left|\lambda\left(x_{1}-\tau y_{1}\right)\right|$, then

$$
\|x+\tau y\|^{2}+\|x-\tau y\|^{2}=\|x+\tau y\|_{2}^{2}+\|x-\tau y\|_{2}^{2}=2\left(1+\tau^{2}\right) .
$$

Ic). If $\|x+\tau y\|_{2} \leq\left|\lambda\left(x_{1}+\tau y_{1}\right)\right|$ and $\|x-\tau y\|_{2}>\left|\lambda\left(x_{1}-\tau y_{1}\right)\right|$, or $\|x+\tau y\|_{2}>$ $\left|\lambda\left(x_{1}+\tau y_{1}\right)\right|$ and $\|x-\tau y\|_{2} \leq\left|\lambda\left(x_{1}-\tau y_{1}\right)\right|$, then

$$
\begin{aligned}
\|x+\tau y\|^{2}+\|x-\tau y\|^{2} & =\lambda^{2}\left(x_{1} \pm \tau y_{1}\right)^{2}+\left(x_{1} \mp \tau y_{1}\right)^{2}+\left(x_{2} \mp \tau y_{2}\right)^{2} \\
& \leq 1+\tau^{2}+\lambda^{2}\left(x_{1}^{2}+\tau^{2} y_{1}^{2}\right)+2 \tau\left(\lambda^{2}-1\right)\left|x_{1} y_{1}\right| \\
& +2 \tau \sqrt{1-x_{1}^{2}} \sqrt{1-y_{1}^{2}} \\
& \leq 2\left(1+\tau^{2}\right)+2 \tau\left(2-\frac{2}{\lambda^{2}}\right)
\end{aligned}
$$

holds by Lemma 2.2. Hence, (2.14)-(2.16) imply (2.13).

Case(II) $1<\lambda \leq \sqrt{2}$. Letting $x=\left(x_{1}, x_{2}\right), y=\left(y_{1}, y_{2}\right)$ again, then (2.14) and (2.15) is also valid. For the third case, first we consider the following function $F(u, v)=\lambda^{2}\left(u^{2}+\tau^{2} v^{2}\right)+2 \tau\left(\lambda^{2}-1\right) u v+2 \tau \sqrt{1-u^{2}} \sqrt{1-v^{2}}$, where $u, v \in\left[0, \frac{1}{\lambda}\right]$. By applying (2.11),(2.12) and (2.7), we have

$$
\begin{aligned}
\frac{1}{2}+\frac{F(0, v)}{2\left(1+\tau^{2}\right)} & =\frac{1}{2}+\frac{\lambda^{2} \tau^{2} v^{2}+2 \tau \sqrt{1-v^{2}}}{2\left(1+\tau^{2}\right)} \leq 2-\frac{1}{\lambda^{2}} \\
\frac{1}{2}+\frac{F(u, 0)}{2\left(1+\tau^{2}\right)} & =\frac{1}{2}+\frac{\lambda^{2} u^{2}+2 \tau \sqrt{1-u^{2}}}{2\left(1+\tau^{2}\right)} \leq 2-\frac{1}{\lambda^{2}} \\
\frac{1}{2}+\frac{F\left(\frac{1}{\lambda}, v\right)}{2\left(1+\tau^{2}\right)} & =\frac{1}{2}+\frac{1+\lambda^{2} \tau^{2} v^{2}+\frac{2 \tau}{\lambda}\left[\left(\lambda^{2}-1\right) v+\sqrt{\lambda^{2}-1} \sqrt{1-v^{2}}\right]}{2\left(1+\tau^{2}\right)} \\
& \leq 2-\frac{1}{\lambda^{2}}
\end{aligned}
$$


and

$$
\begin{aligned}
\frac{1}{2}+\frac{F\left(u, \frac{1}{\lambda}\right)}{2\left(1+\tau^{2}\right)} & =\frac{1}{2}+\frac{\tau^{2}+\lambda^{2} u^{2}+\frac{2 \tau}{\lambda}\left[\left(\lambda^{2}-1\right) u+\sqrt{\lambda^{2}-1} \sqrt{1-u^{2}}\right]}{2\left(1+\tau^{2}\right)} \\
& \leq \frac{1}{2}+\frac{1+\lambda^{2} \tau^{2} u^{2}+\frac{2 \tau}{\lambda}\left[\left(\lambda^{2}-1\right) u+\sqrt{\lambda^{2}-1} \sqrt{1-u^{2}}\right]}{2\left(1+\tau^{2}\right)} \\
& \leq 2-\frac{1}{\lambda^{2}}
\end{aligned}
$$

Hence, by applying (2.17)-(2.18) and Lemma 2.3, we have that

$$
\begin{aligned}
& \frac{\|x+\tau y\|^{2}+\|x-\tau y\|^{2}}{2\left(1+\tau^{2}\right)} \\
& \leq \frac{1}{2}+\frac{F\left(\left|x_{1}\right|, \mid y_{1}\right) \mid}{2\left(1+\tau^{2}\right)} \\
& \leq \frac{1}{2}+\max \left\{\frac{F(u, v)}{2\left(1+\tau^{2}\right)} ;(u, v) \in \partial\left[0, \frac{1}{\lambda}\right] \times\left[0, \frac{1}{\lambda}\right]\right\} \\
& \leq 2-\frac{1}{\lambda^{2}} .
\end{aligned}
$$

Therefore, (2.13) is valid. Whence (2.13) and (1.1) imply $C_{N J}\left(\mathbb{R}_{\lambda}^{2}\right) \leq 2-\frac{1}{\lambda^{2}}$. On the other hand, if taking $x=\left(\frac{1}{\lambda}, \sqrt{1-\frac{1}{\lambda^{2}}}\right), y=\left(\frac{1}{\lambda},-\sqrt{1-\frac{1}{\lambda^{2}}}\right)$, we have

$$
C_{N J}\left(\mathbb{R}_{\lambda}^{2}\right) \geq \frac{\|x+y\|^{2}+\|x-y\|^{2}}{4}=2-\frac{1}{\lambda^{2}},
$$

which completes the proof of Theorem 2.1.

Corollary 2.7. If $1 \leq \lambda<1+\frac{\sqrt{3}}{3}$, then Banaś-Fraczek space $\mathbb{R}_{\lambda}^{2}$ and its dual space have uniform normal structure.

Proof. Because $1 \leq \lambda<1+\frac{\sqrt{3}}{3}$ implies

$$
C_{N J}\left(\mathbb{R}_{\lambda}^{2}\right) \leq 2-\frac{1}{\lambda^{2}}<\frac{1+\sqrt{3}}{2}
$$

Hence, by a Satit Saejung's result (see [5]), we complete the proof of Corollary 2.7 .

Acknowledgement. We express our cordial thanks to the referees. This work is supported by the National Natural Science Foundation of China(Nos. 11271112; 11201127) and Technology and Pioneering project in Henan province (No.122300410110). 


\section{REFERENCES}

1. J. Banaś and K. Frączek, Deformation of Banach spaces, Comment. Math. Univ. Carolinae 34 (1993), no. 1, 47-53.

2. J.A. Clarkson, The von Neumann-Jordan constant for the Lebesgue space, Ann. of Math. 38 (1937), no. 1, 114-115.

3. J. Gao and K.S. Lau, On two classes of Banach spaces with uniform normal structure, Studia Math. 99 (1991), no. 1, 41-56.

4. M. Kato, L. Maligranda and Y. Takahashi, On James and Jordan-von Neumann constants and the normal structure coefficient of Banach spaces, Studia Math. 144 (2001), no. 3, $275-295$.

5. S. Saejung, On James and von Neumann-Jordan constants and sufficient conditions for the fixed point property, J. Math. Anal. Appl. 323 (2006), no. 2, 1018-1024.

6. Y. Takahashi and M. Kato, A simple inequality for the von Neumann-Jordan and James constants of a Banach spaces, J. Math. Anal. Appl. 359 (2009), no. 2, 602-609.

7. F. Wang, On the James and von Neumann-Jordan constants in banach spaces, Proc. Amer. Math. Soc, 138 (2010), no. 2, 695-701.

8. C. Yang and H. Li, An inequality between Jordan-von Neumann constant and James constant, Appl. Math. Lett. 23 (2010), no. 3, 277-281.

9. C. Yang and F. Wang, On a new geometric constant related to the von Neumann-Jordan constant, J. Math. Anal. Appl. 324 (2006), no. 1, 555-565.

College of Mathematics and Information Science, University of Henan Normal, Xinxiang 453007, Henan, P.R.China.

E-mail address: yangchangsen0991@sina.com 